\title{
High-Volume Lesions Using a New Second-Generation Open Irrigation Radiofrequency Catheter Are Associated with the Development of Inhomogeneous Lesions
}

\author{
FERDI AKCA, B.Sc., * MARTHA HUBAY, M.D., Ph.D.,† ENDRE ZIMA, M.D., Ph.D.,† \\ GÁBOR SZÉPLAKI, M.D.,† ESZTER M. VÉGH, PH.D.,† JUDIT SKOPÁL, PH.D.,† \\ ZSUZSANNA LENDVAI,† DOMINIC THEUNS, PH.D., * BELA MERKELY, M.D., PH.D.,† \\ and TAMAS SZILI-TOROK, M.D., PH.D.* \\ From the *Erasmus Medical Center, Rotterdam, The Netherlands; and +Heart Centre, Semmelweis University, \\ Budapest, Hungary
}

Background: After catheter ablation there is often a discrepancy between acute and chronic success rates. We aimed to evaluate major determinants for lesion quality and understand different manifestations of lesion structures.

Methods: In a canine thigh muscle model radiofrequency (RF) current was delivered for 60 seconds at $30 \mathrm{~W}(\mathrm{n}=39)$ or $50 \mathrm{~W}(\mathrm{n}=18)$ with 15 -g contact force. A second-generation 12-hole gold open irrigation catheter (SGIT) and a first-generation six-hole platinum-iridium catheter (FGIT; Biotronik, Berlin, Germany) were used. Electrode and tissue temperatures (at the surface and 3.5-mm and 7-mm depth) were recorded and lesion dimensions were measured. Lesions with steam pops were excluded. Histological examination was performed to evaluate homogeneity of the lesions. Inhomogeneity was defined as a visual multiband lesion pattern indicating different histological characteristics.

Results: In total 57 lesions were created. Seventeen lesions were excluded (steam pops) and 40 lesions were analyzed. A total number of 11 homogeneous and 29 inhomogeneous lesions were identified. Using the SGIT catheter $16.7 \%$ of the lesions was homogeneous and $83.3 \%$ inhomogeneous; for FGIT it was $43.8 \%$ and $56.2 \%(P=0.065)$, respectively. Homogeneous lesions had lower volumes as compared to inhomogeneous lesions (514.0 \pm 198.8 vs $914.8 \pm 399.1 \mathrm{~mm}, P=0.003$ ). Multiple logistic regression analysis indicated that the SGIT catheter is a significant predictor for inhomogeneous lesions (odds ratio 6.5, 95\% confidence interval 1.1-38.8; $P=0.040$ ) independent from power setting and flow rate.

Conclusions: The development of inhomogeneous lesions after acute RF ablation is associated with higher lesion volumes and the use of the second-generation irrigation gold-tip catheter. (PACE 2014; 37:864-873)

catheter ablation, gold-tip catheter, platinum-iridium-tip catheter, histology, lesion formation

\section{Introduction}

The use of irrigated-tip radiofrequency (RF) catheters is a well-established therapeutic option that is implemented in everyday catheter ablation procedures. ${ }^{1}$ It has been demonstrated that it increases efficacy of catheter ablation procedures

\footnotetext{
This study was funded by Biotronik (Berlin, Germany).

Tamas Szili-Torok and Bela Merkely are consultants for Biotronik.

The other authors have no disclosures.
}

Address for reprints: Tamas Szili-Torok, M.D., PH.D., Thoraxcenter, Department of Clinical Electrophysiology, Erasmus MC Postbus 2040, 3000 CA Rotterdam, The Netherlands. Fax: 3110-703-4420; e-mail: t.szilitorok@erasmusmc.nl

Received July 25, 2013; revised December 13, 2013; accepted December 13, 2013.

doi: 10.1111/pace.12359

C)2014 Wiley Periodicals, Inc. and allows for increased energy delivery and higher lesion volumes. ${ }^{2}$ Several catheter designs are currently available using either a gold ( $\mathrm{Au}$ )-tip or a platinum iridium (PtIr)-tip electrode. Multiple studies have compared these irrigated-tip RF catheters and evaluated several parameters such as catheter temperatures and lesion volumes. ${ }^{3-5}$ The irrigated $\mathrm{Au}$-tip electrode has been well evaluated, which allows for improved energy delivery at lower catheter-tip temperatures as compared to irrigated PtIr-tip catheters. ${ }^{6,7}$ However, about the new-generation 12-holes RF irrigation catheter only limited data are available. ${ }^{8}$ Previous studies on open irrigation RF ablation catheters focused on created lesion volumes by using the same formula, which was the basis of the comparison. ${ }^{9}$ For multipolar ablation catheters even lesion continuity was studied, ${ }^{10}$ but prior studies never meticulously evaluated the structure of the lesions. 


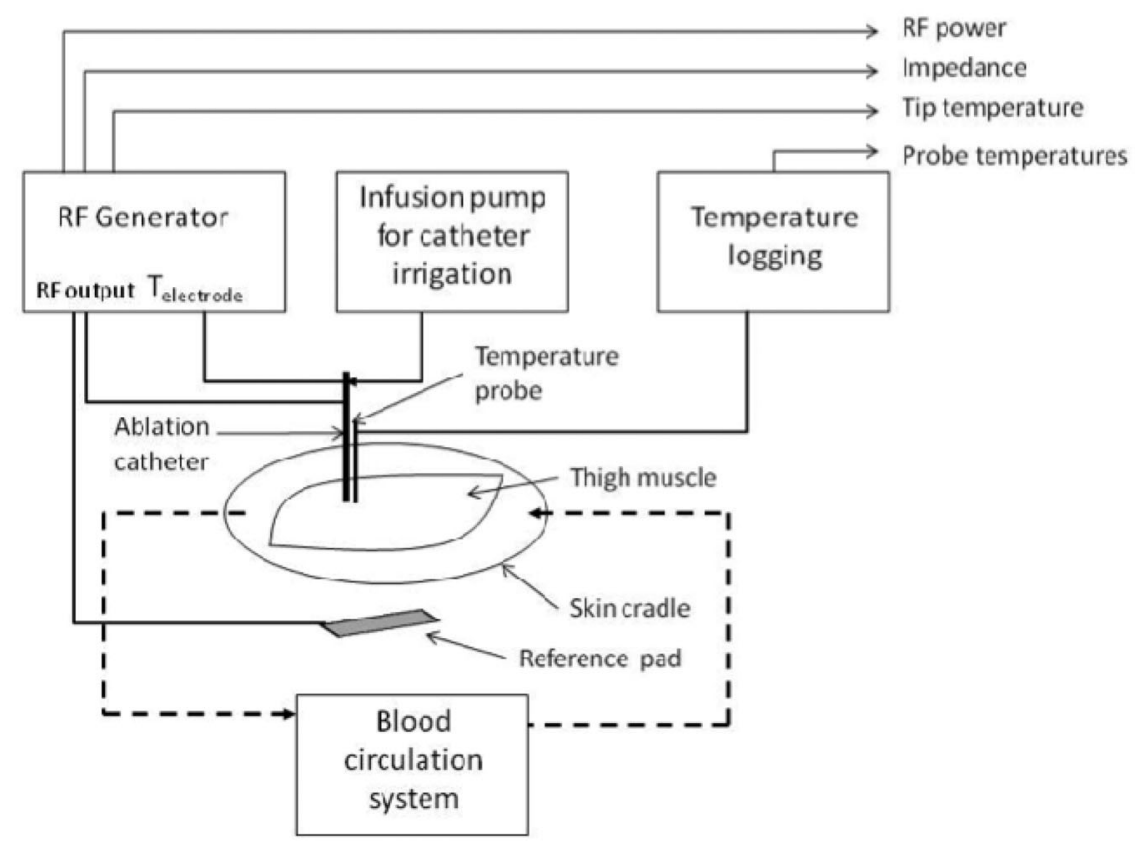

Figure 1. Experimental setup. Schematic representation of the experimental setup. The reference pad is attached to the contra-lateral thigh.

Energy delivery of the ablation catheter is dependent on multiple factors such as contact force, irrigation flow rate, power output, ablation time, ablated tissue characteristics, catheter tip orientation, and the type of metal from which the catheter tip is manufactured. ${ }^{11}$ These individual factors influence the efficacy of energy delivery to the tissue and therefore contribute to lesion formation and lesion quality. Different conducting properties of the ablation catheter and different amounts of absorbed energy by the tissue might lead to different macroscopic and microscopic manifestations of the lesion (coagulation necrosis and reactive degeneration with interstitial edema). This could effect chronic lesion development. Our aim was to assess which characteristics can play a role as major determinants for lesion quality and to understand the different manifestations of lesion structures. Furthermore, we aimed to evaluate whether the use of a first-generation (FGIT) and second-generation (SGIT) irrigated-tip RF catheter has effect on the development of lesion size and structures. Our primary hypothesis was that the use of different irrigation catheters (firstand second-generation) would result in different lesion sizes and macroscopic and microscopic lesion characteristics.

\section{Description of the Model}

\section{Methods}

In this study, a canine thigh muscle model has been used. This model was developed and first described by Nakagawa and colleagues. ${ }^{12}$ This study has been conducted according to good laboratory practice regulations in a certified laboratory. Three mongrel dogs were anesthetized with sodium pentobarbital $(25 \mathrm{mg} / \mathrm{kg})$ and either mechanically ventilated with room air or allowed to breathe spontaneously. General anesthesia is maintained with additional doses of sodium pentobarbital. The arterial pressure is continuously monitored by means of a right carotid artery cannulation. A skin incision was made and the skin edges were raised to create a cradle. The cradle was filled with blood (controlled $37^{\circ} \mathrm{C}$, activated clotting time $>350$ seconds), which is exchanged at a rate of $250 \mathrm{~mL} / \mathrm{min}$, to mimic cardiac circulation. The ablation electrode was positioned in perpendicular or parallel orientation with respect to the muscle surface and held at a constant contact force of $15 \mathrm{~g}$ by use of a custommade balance. This amount of contact force was applied, since this value represents the average amount of contact force that is applied mostly by operators during pulmonary vein isolation (PVI) and is therefore a clinically relevant value. ${ }^{13}$

Temperatures were measured by fluoroptic temperature probes (Luxtron model STB, Santa Clara, CA, USA) immediately below the surface and as close as possible to the ablation catheter, as an approximation of the electrode-tissue interface temperature, and at $3.5 \mathrm{~mm}$ and $7 \mathrm{~mm}$ below the muscle surface. All the temperatures were logged 


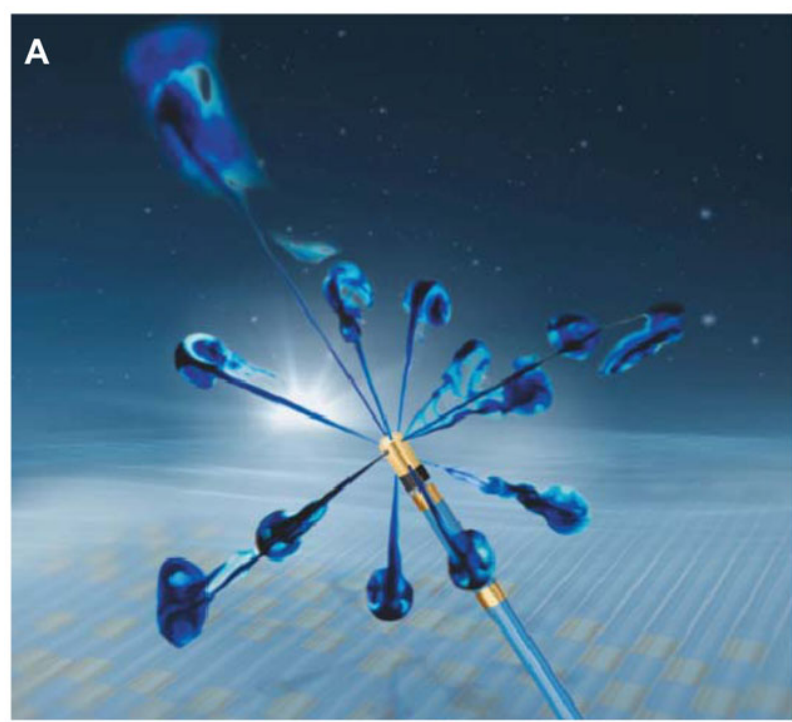

B

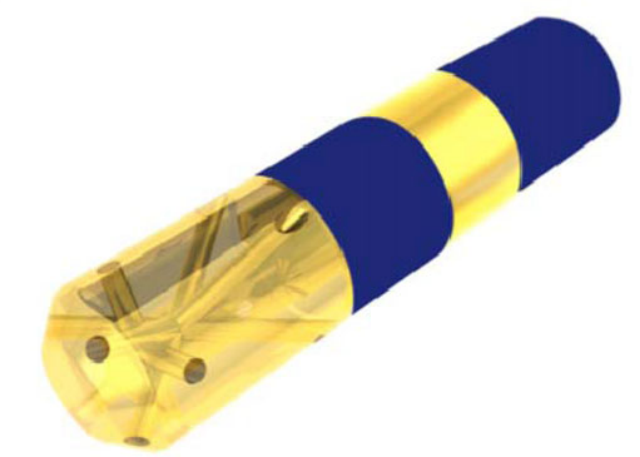

Figure 2. Catheter designs. Image of the novel 12-hole $A u$ open-irrigation catheter displaying the irrigation directions (A) and the new catheter design (B).

electronically and the maximum and mean temperatures were recorded. Additionally, the data from the RF generator, including the programmed and actually delivered RF power, impedance, and electrode temperature, were electronically recorded (Fig. 1).

\section{Ablation Catheters}

$\mathrm{RF}$ ablations were carried out using either the (SGIT) AlCath Flux eXtra Gold catheter (Biotronik, Berlin, Germany) or the (FGIT) AlCath Flux Full Circle Pt/Ir catheter (Biotronik). Both catheters are $7 \mathrm{~F}$ in diameter and have a $3.5-\mathrm{mm}$ ablation-tip electrode. The SGIT ablation catheter has an Au ablation electrode with a novel configuration of 12 irrigation holes (Fig. 2). The FGIT catheter has a platinum-iridium electrode with six irrigation holes.

\section{Ablation Protocol}

Lesions were created with the ablation catheter oriented parallel and perpendicular to the muscle surface; an RF power of $30 \mathrm{~W}$ and $50 \mathrm{~W}$; and $8 \mathrm{~mL} / \mathrm{min}, 15 \mathrm{~mL} / \mathrm{min}$, and $30 \mathrm{~mL} / \mathrm{min}$ irrigation flow rates. The ablation catheters were irrigated through the catheter lumen with heparinized $(2 \mathrm{U} / \mathrm{mL})$ normal saline at room temperature $(20$ $22^{\circ} \mathrm{C}$ ). Ablation settings (RF power, irrigation flow rate) and conditions (catheter type and electrode orientation) were applied in random order to avoid bias using the randomization function in an Excel ${ }^{\circledR}$ spreadsheet (v2010 Microsoft Corp., Redmond, WA, USA). The randomization was carried out according to the following procedure: randomization of the power output and the electrode orientation (perpendicular or parallel); for each orientation the type of catheter was randomized, and for each combination of catheter type and electrode orientation the irrigation flow rate was randomized. For each lesion, RF energy was delivered for 60 seconds or until the first steam pop. The occurrence of steam pops was recorded and these lesions were excluded from histological assessment since it could affect lesion dimensions and macro- and microscopic characteristics of the tissue.

\section{Tissue Staining and Conservation}

Two hours after completion of the RF ablations at the left and right thigh muscle, 30 $\mathrm{mL}$ of $2 \%$ triphenyl tetrazolium chloride was administered intravenously as a staining allowing macroscopic visualization of necrotic tissue and revealing the extent of the lesion (Fig. 3). After sacrificing the dog, thigh muscle dimensions were determined before fixation to correct tissue shrinkage due to fixation. Following formaldehyde fixation, the lesions were sliced into $1-$ to $2-\mathrm{mm}$ thin slices and the lesion dimensions were determined as described by Lewalter and colleagues. ${ }^{14}$ Afterwards, the muscle slices were embedded into paraffin and sections were prepared and stained according to the phosphotungstic acidhematoxylin (PTAH) method to demonstrate muscle structure. This allowed microscopic evaluation of the lesions (Figs. 4 and 5).

\section{Lesion Dimensions}

For each created lesion, the following dimensions were determined histologically: lesion depth (A), maximum lesion diameter (B), lesion depth at maximum diameter $(\mathrm{C})$, and lesion diameter at surface (D). The lesion volume $\left(\mathrm{V}_{\text {lesion }}\right)$ was calculated using the following formula ${ }^{9}$ :

$$
\mathrm{V}_{\text {lesion }}=(1 / 6) \times \pi \times\left(\mathrm{A} \times \mathrm{B}^{2}+\mathrm{C} \times \mathrm{D}^{2} / 2\right) .
$$




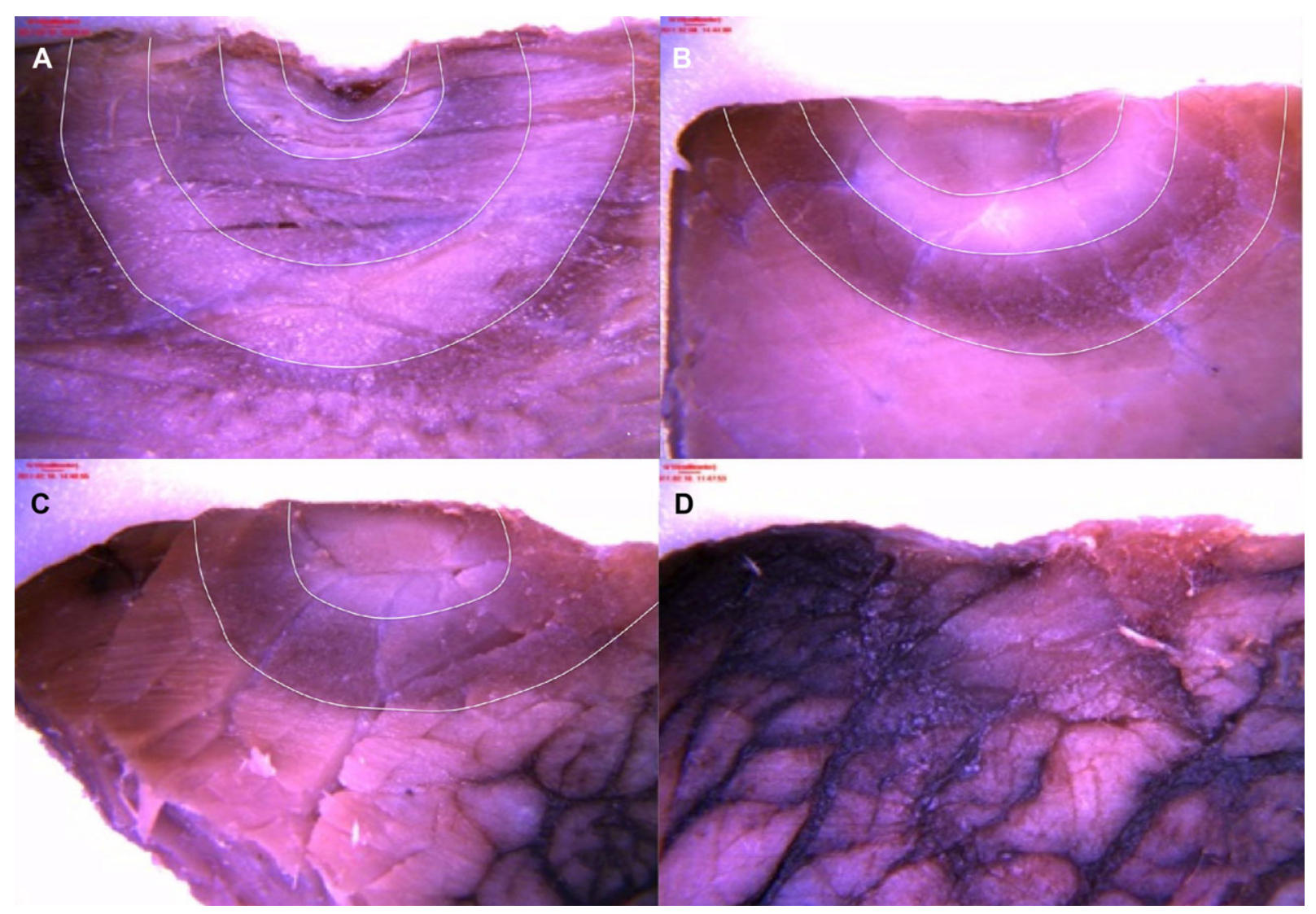

Figure 3. Macroscopic image of the fixated lesion tissues. Lesion A illustrates a lesion with four macroscopic bands, lesion $B$ demonstrates three bands, lesion $C$ has two macroscopic bands, and during creation of lesion $D$ steam pop occurred.

Muscle dimensions before and after fixation by formalin, allowing for correction with respect to tissue shrinkage, were not obtained for all experiments. Therefore, uncorrected results, directly obtained from fixated muscle tissue, were used to calculate mean lesion dimensions. For those experiments in which shrinkage due to fixation could be determined, muscle shrinkage was most frequently less than $5 \%$.

\section{Lesion Quality Assessment}

After staining the tissue, macroscopic and histological examination of the lesions were performed. The macroscopic examination allowed evaluation of the homogeneity of the RF lesions. This was determined by assessing the number of different colored bands that were visible in the lesion, indicating different histological characteristics (Figs. 4 and 5). These macroscopic classifications were confirmed histologically. A homogenous lesion was defined as an RF lesion in which a maximum of two different colored bands were observed. Inhomogeneous lesions were lesions with a pattern of more than two visual bands. Two authors (F.A. and T.S.T.) independently classified the lesions according to the amount of visible bands. In case of a discrepant classification, this lesion was re-evaluated to gain a consensus opinion. Between the homogenous and inhomogeneous lesions analyses were performed to determine in what extend catheter type, irrigation flow rate, catheter orientation, and power settings are responsible for the different lesion formation.

\section{Statistics}

Normality of distribution was determined by using the Kolmogorov-Smirnov test. Continuous variables were expressed as mean \pm standard deviation, if normally distributed, and compared with the Student's $t$-test for independent samples. In case of nonnormal distribution of data, medians and interquartile ranges (IQRs) were reported and the Mann-Whitney U test was used for data comparison. Categorical data were expressed as percentages and compared with the $\chi^{2}$ test or 


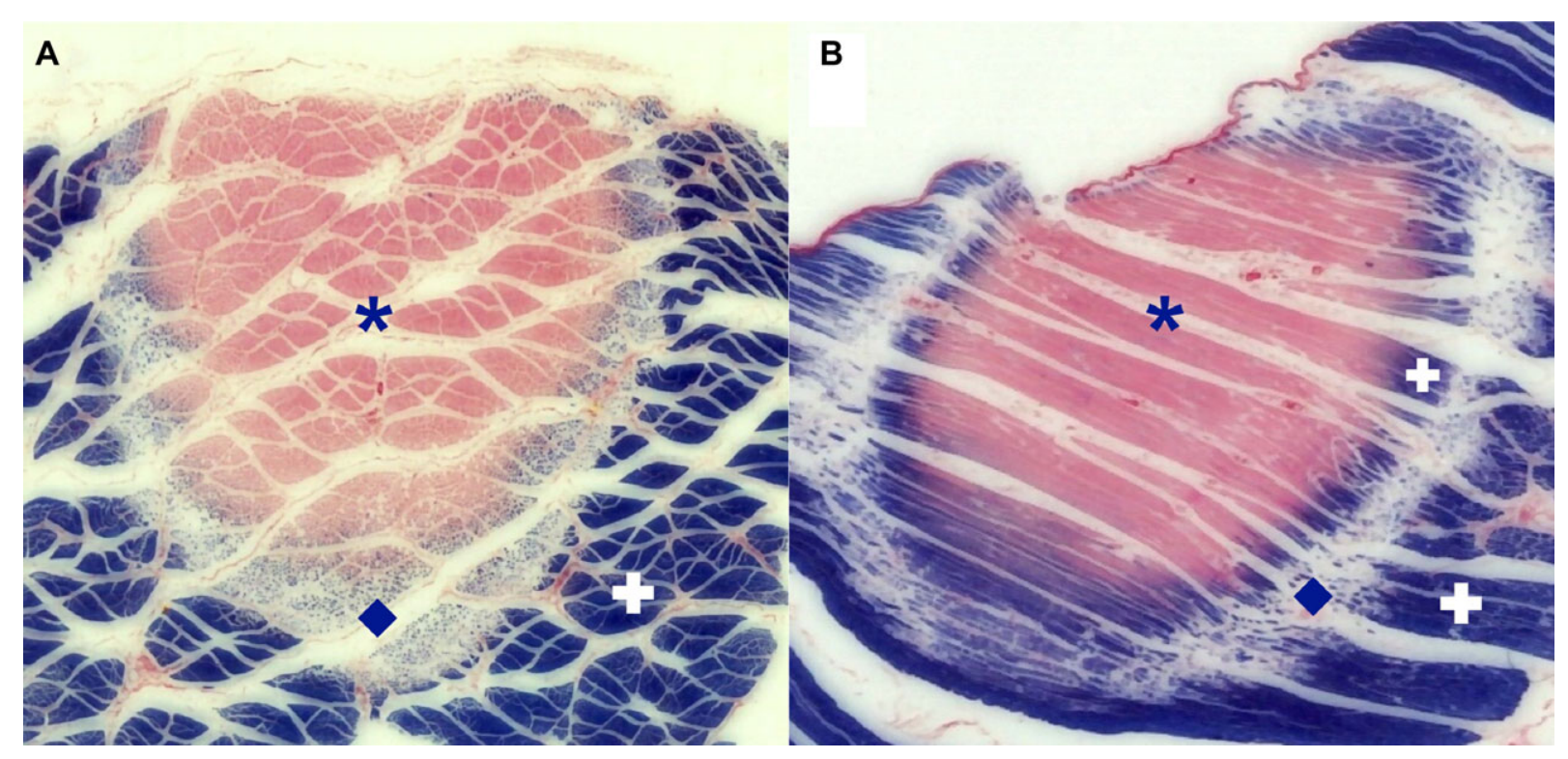

Figure 4. Two histological phosphotungstic acid-hematoxylin (PTAH)-stained pictures of the lesions illustrating different microscopic bands. (A) Overview of a lesion with two lesion bands. (B) Overview of a lesion with three lesion bands. * Central coagulation necrosis; Reactive zone with hypercontraction, interstitial edema, and degenerative reaction of the muscle cells. + Intact muscle.

Fisher's exact test when appropriate. Univariate analyses were performed for all variables and odds ratios (ORs) and 95\% confidence intervals (95\% CIs) were determined. All variables with $\mathrm{P}<0.150$ in the univariate analysis (due to the limited sample size) were entered into a multivariate logistic regression model. ORs, 95\% CIs, and P-values were calculated. Because of the observational nature of the study, power calculation to prove statistical significant differences has not been performed. Statistical analysis was performed using SPSS 15.0 (IBM Corp., Armonk, NY, USA). Statistical significance was defined as $\mathrm{P}<0.05$ (two-tailed).

\section{Results}

\section{Lesion Overview}

Using the mongrel dog model, 57 lesions were created. Seventeen out of 57 lesions were not further analyzed due to the occurrence of steam pops. In $23.5 \%$ of the cases the steam pop occurred using the SGIT catheter and in $76.5 \%$ the FGIT catheter was used (OR $0.2,95 \%$ CI $0.1-0.7 ; \mathrm{P}=$ 0.012). No differences were found between the SGIT and FGIT catheter for power and flow rate setting $(50.0 \pm 0.0$ vs $43.9 \pm 9.6 \mathrm{~W}, \mathrm{P}=0.230$; $15.0 \pm 0.0$ vs $16.9 \pm 8.1 \mathrm{~mL} / \mathrm{min}, \mathrm{P}=0.660$, respectively). In total, 40 lesions were further analyzed in this study.

\section{Homogeneous and Inhomogeneous Lesions}

Eleven lesions $(27.5 \%)$ were classified as a homogenous lesion and 29 lesions $(72.5 \%)$ had an inhomogeneous aspect. In total, one lesion had a single visual band (2.5\%), 10 lesions had two bands $(25.0 \%), 26$ lesions had three bands $(65.0 \%)$, and three lesions had four bands $(7.5 \%)$. In Table I the distribution of catheter type, orientation, power output, and flow rate is displayed between the homogeneous and inhomogeneous group. For all these variables, an equal distribution was observed between the two groups (Table I). Using the SGIT catheter $16.7 \%$ of the lesions was homogeneous and $83.3 \%$ was inhomogeneous (OR $0.3,95 \%$ CI $0.1-1.1 ; \mathrm{P}=0.065)$. The FGIT catheter created in $43.8 \%$ homogeneous lesions and in $56.2 \%$ inhomogeneous lesions were formed (OR 3.9 , 95\% CI 0.9-16.7; $\mathrm{P}=0.065)$. The median number of colored bands in the homogeneous group was 2 (IQR [2-2]) and 3 (IQR [3-3]) bands were evident in the inhomogeneous group $(\mathrm{P}<$ 0.001 ).

\section{Histological Tissue Assessment}

Histological evaluation demonstrated clearly a central area of the lesions, which stains light pinkish using PTAH staining and illustrates coagulation necrosis (Fig. 5). Between the central area and the intact muscle, there is a reactive zone, with mild damaged muscle tissue, hypercontraction, interstitial edema, and slightly decreased staining. 


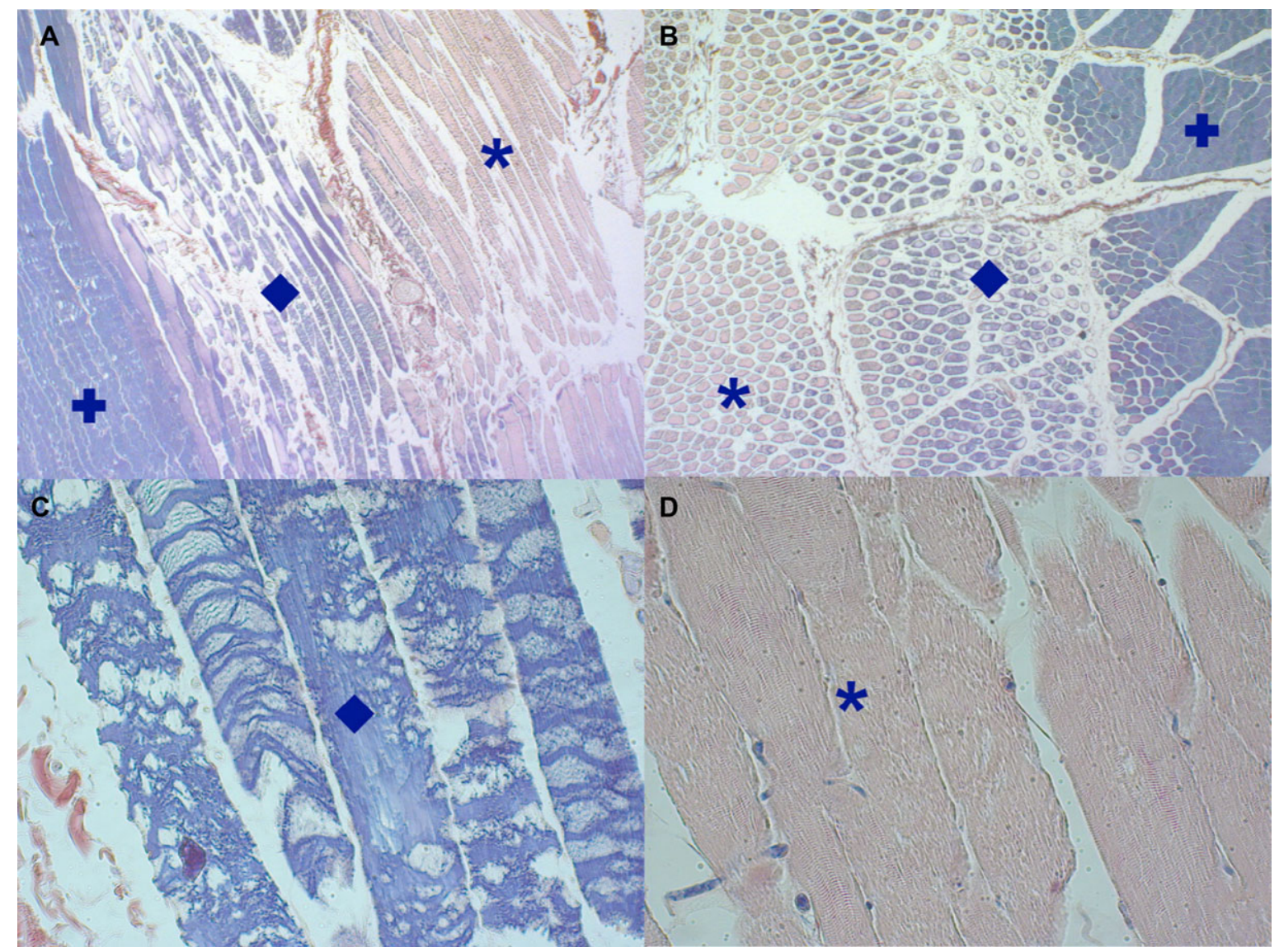

Figure 5. Histological illustrations of the lesions using phosphotungstic acid-hematoxylin (PTAH) staining. (A) Overview of the lesion border (magnification $4 \times$, longitudinal section). (B) Overview of the lesion border (magnification $4 \times$, cross-section). (C) Border zone of the lesion (magnification $40 \times$ ). (D) Central coagulation necrosis (magnification $40 \times)$. * Central coagulation necrosis; $\diamond$ Reactive zone with hypercontraction, interstitial edema, and degenerative reaction of the muscle cells. + Intact muscle.

Intact muscle tissue stained blue-purple and was visible either at the border of the lesion or between the central coagulation necrosis and reactive zone (Fig. 4).

\section{Lesion Dimensions, Temperatures, and Power Delivery}

In Table II the lesion dimensions and recorded data during ablation are presented. Homogeneous lesions were less deep and had a smaller maximum lesion diameter as compared to inhomogeneous lesions $(6.2 \pm 1.5$ vs $7.9 \pm 1.4 \mathrm{~mm}$, $\mathrm{P}=0.003 ; 12.3 \pm 1.8$ vs $14.2 \pm 2.1, \mathrm{P}=0.011$, respectively). This resulted in significantly lower lesion volumes for homogeneous lesions (514.0 \pm 198.8 vs $914.8 \pm 399.1 \mathrm{~mm}, \mathrm{P}=0.003)$. The SGIT catheter is associated with larger lesion volumes as compared to the FGIT catheter ( $912.8 \pm 438.6$ vs $624.2 \pm 234.7 \mathrm{~mm}, \mathrm{P}=0.025)$. In Figure 6 the mean lesion volumes for each number of observed bands is illustrated. No differences were observed for the mean and maximum electrode, interface, and tissue temperatures between the homogeneous and inhomogeneous group (Table II). However, there is a trend that inhomogeneous lesions occur more frequently using the $30 \mathrm{~W}$ power setting than with the $50 \mathrm{~W}$ power output but this could not be demonstrated with statistical significance $(77.1 \%$ vs $40.0 \%, \mathrm{P}=0.117$ ).

\section{Multiple Logistic Regression Model}

Multiple logistic regression analysis was performed to determine independent predictors for inhomogeneous lesion formation. Catheter type, power setting, and $8 \mathrm{~mL} / \mathrm{min}$ flow rate setting were entered into the model since univariate analysis showed a P-value $<0.150$. The results indicate that the use of the SGIT catheter (OR $6.5,95 \%$ CI $1.1-38.8 ; \mathrm{P}=0.040$ ) is a statistically significant predictor for inhomogeneous lesion 
AKCA, ET AL.

Table I.

Distribution of Ablation Settings

\begin{tabular}{|c|c|c|c|c|c|c|c|c|}
\hline Variable & Homogeneous Lesion & $\mathbf{N}$ & Inhomogeneous Lesion & $\mathbf{N}$ & Total & OR & $95 \% \mathrm{Cl}$ & P-Value \\
\hline Au† catheter & $16.7 \%$ & 4 & $83.3 \%$ & 20 & 24 & 0.3 & $0.1-1.1$ & 0.065 \\
\hline PtIr $\ddagger$ catheter & $43.8 \%$ & 7 & $56.2 \%$ & 9 & 16 & 3.9 & $0.9-16.7$ & \\
\hline Parallel orientation & $31.6 \%$ & 6 & $68.4 \%$ & 13 & 19 & 1.5 & $0.4-6.0$ & 0.422 \\
\hline Perpendicular orientation & $23.8 \%$ & 5 & $76.2 \%$ & 16 & 21 & 0.8 & $0.2-3.4$ & \\
\hline Power $30 \mathrm{~W}$ & $22.9 \%$ & 8 & $77.1 \%$ & 27 & 35 & 0.2 & $0.0-1.4$ & 0.117 \\
\hline Power $50 \mathrm{~W}$ & $60.0 \%$ & 3 & $40.0 \%$ & 2 & 5 & 5.1 & $0.7-35.8$ & \\
\hline Flow rate $8 \mathrm{~mL} / \mathrm{min}$ & $12.5 \%$ & 2 & $87.5 \%$ & 14 & 16 & 0.2 & $0.0-1.3$ & 0.083 \\
\hline Flow rate $15 \mathrm{~mL} / \mathrm{min}$ & $36.4 \%$ & 8 & $63.6 \%$ & 14 & 22 & 2.9 & $0.6-13.0$ & 0.151 \\
\hline Flow rate $30 \mathrm{~mL} / \mathrm{min}$ & $50.0 \%$ & 1 & $50.0 \%$ & 1 & 2 & 2.8 & $0.2-49.1$ & 0.479 \\
\hline
\end{tabular}

†Gold.

¥Platinum-iridium.

The data are presented as percentage, odds ratio (OR), 95\% confidence interval ( $\mathrm{Cl}$ ), and P-value.

Table II.

Lesion Dimensions and Recorded Data during Ablation

\begin{tabular}{lccc}
\hline Variable & Homogeneous Lesion & Inhomogeneous Lesion & P-Value \\
\hline Lesion depth $(\mathrm{mm})$ & $6.2 \pm 1.5$ & $7.9 \pm 1.4$ & 0.003 \\
Maximum lesion diameter $(\mathrm{mm})$ & $12.3 \pm 1.8$ & $14.2 \pm 2.1$ & 0.011 \\
Lesion depth at maximum diameter $(\mathrm{mm})$ & $0.0[0.0-0.0]$ & $0.0[0.0-3.3]$ & 0.218 \\
Lesion diameter at surface $(\mathrm{mm})$ & $11.6 \pm 2.0$ & $13.0 \pm 2.5$ & 0.116 \\
Volume $(\mathrm{mm})$ & $514.0 \pm 198.8$ & $914.8 \pm 399.1$ & 0.003 \\
Mean $\mathrm{T}_{\text {electrode }}\left({ }^{\circ} \mathrm{C}\right)$ & $39.8 \pm 7.1$ & $39.7 \pm 6.2$ & 0.988 \\
Mean $\mathrm{T}_{\text {interface }}\left({ }^{\circ} \mathrm{C}\right)$ & $46.6[39.1-61.8]$ & $44.7[38.9-57.9]$ & 0.720 \\
Mean $\mathrm{T}_{3.5 \mathrm{~mm}}\left({ }^{\circ} \mathrm{C}\right)$ & $40.9[36.7-44.4]$ & $39.3[38.0-45.6]$ & 0.788 \\
Mean $\mathrm{T}_{7.0 \mathrm{~mm}}\left({ }^{\circ} \mathrm{C}\right)$ & $41.3[36.7-42.3]$ & $38.8[36.2-42.0]$ & 0.591 \\
Maximum $\mathrm{T}_{\text {electrode }}\left({ }^{\circ} \mathrm{C}\right)$ & $41.7[37.8-46.0]$ & $38.6[37.1-48.1]$ & 0.570 \\
Maximum $\mathrm{T}_{\text {interface }}\left({ }^{\circ} \mathrm{C}\right)$ & $61.0 \pm 21.1$ & $57.8 \pm 17.9$ & 0.638 \\
Maximum $\mathrm{T}_{3.5 \mathrm{~mm}}\left({ }^{\circ} \mathrm{C}\right)$ & $46.2[37.8-49.9]$ & $44.1[39.1-52.9]$ & 0.765 \\
Maximum $\mathrm{T}_{7.0 \mathrm{~mm}}\left({ }^{\circ} \mathrm{C}\right)$ & $49.0 \pm 18.2$ & $44.0 \pm 7.3$ & 0.216 \\
Mean Power Delivery $(\mathrm{W})$ & $34.7 \pm 8.9$ & $30.1 \pm 3.7$ & 0.024 \\
Mean Impedance $(\Omega)$ & $84.6 \pm 8.6$ & $84.4 \pm 6.3$ & 0.922 \\
\hline
\end{tabular}

The data are presented in mean \pm standard deviation or median (interquartile range) and $\mathrm{P}$-value.

formation independent from power setting (OR $0.1,95 \%$ CI $0.0-1.5 ; \mathrm{P}=0.107)$ and flow rate (OR $3.1,95 \%$ CI $0.5-20.5 ; \mathrm{P}=0.235$ ).

\section{Discussion}

The main findings of this study are (1) that inhomogeneous lesions are frequent entities that occur often with RF catheter ablation, (2) the occurrence of inhomogeneous lesions is associated with higher lesion volumes and is not related to tissue temperature, and (3) the use of the second-generation irrigated Au-tip catheter is an independent predictor for inhomogeneous lesion development.

\section{Inhomogeneous Lesions}

Lesion structures with a visible multiband pattern, which indicated an inhomogeneous lesion structure, are more prone to occur with the SGIT catheter. Using multiple logistic regression we demonstrated that the use of this new catheter, independent from power output and irrigation flow rate, is associated with a higher incidence of inhomogeneous lesion development. An important question arises: what are the 


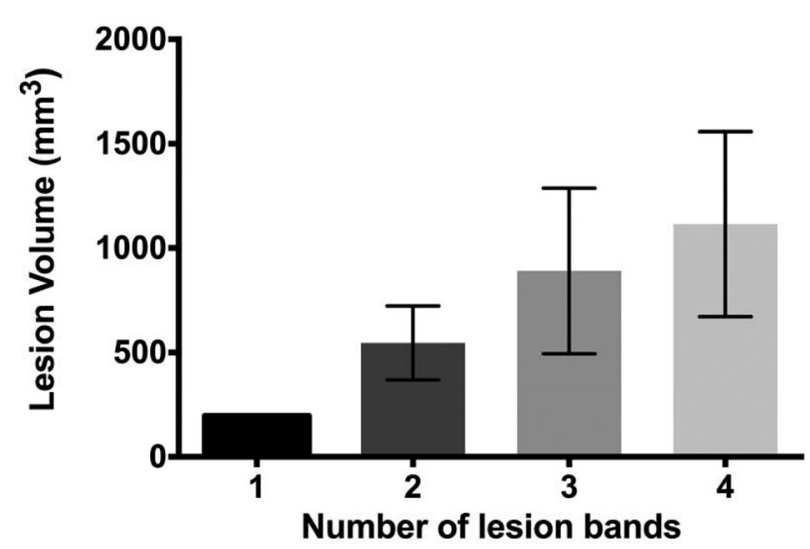

Figure 6. Lesion volumes presented for each number of observed bands. The mean values \pm standard deviation of the lesion volumes are presented. Four categories of bands were observed: lesions with one band $(n=1)$, two bands $(n=10)$, three bands $(n=26)$, and four bands $(n=3)$.

possible underlying mechanisms of these findings, since multiple factors are important determinants for lesion formation and could contribute to these results? Contact force of the catheter with the tissue, irrigation, power output, and catheter design (including irrigation design and metal type) are important factors that influence lesion development. ${ }^{11}$ Weiss and colleagues ${ }^{15}$ demonstrated that irrigation has important effects on lesion formation. The use of irrigation allows operators to safely prolong ablation time and increase power output to create higher lesion volumes. ${ }^{12}$ Another factor that influences lesion formation is the type of metal from the catheter tip. Lewalter and colleagues ${ }^{14}$ compared $\mathrm{Au}$ and PtIr nonirrigated-tip catheters and found that the $\mathrm{Au}$-tip electrode is associated with deeper lesions, which is in line with our findings. The increased lesion volumes using Au-tip electrode are thought to be related to the superior thermal conductive properties of the metal as compared to PtIr. The thermoconductivity of $\mathrm{Au}$ is four times better than of the PtIr alloy. This allows improved and more efficient energy delivery to the tissue and therefore larger lesions. ${ }^{4}$

However, the manifestation of inhomogeneous lesions could not be attributed to one aspect alone. A theory in which multiple determinants of lesion formation are involved could provide an answer about the existence of homogeneous and inhomogeneous lesion structures. The SGIT catheter, with its novel irrigation design, allows for better cooling of the surrounding tissue and therefore less temperature increase. ${ }^{12}$ The additional proximal irrigation holes of the SGIT catheter may lead to more homogeneous tissue cooling over a larger area in a parallel orientation specifically. However, with perpendicular catheter orientation, the proximal holes of the SGIT catheter reduce saline flow out of the distal holes and consequently tissue cooling can be reduced as compared to FGIT. Furthermore, the amount of saline irrigation determines the amount of cooling at the tissue surface. Nakagawa et al. $^{12}$ described competing effects of heating from the underlying tissue with the cooling from the saline irrigation. This eventually leads to lesion development several millimeters below the surface instead of the thin layer of myocardium surrounding the catheter. The differences in irrigation efficacy between the two catheter types could lead to lesion formation at different depths in the tissue. This "indirect" heating and the differences in "direct" heating at the muscle surface due to the novel catheter design could lead an inhomogeneous lesion structure as has been described in this study. When these factors of the SGIT catheter are combined (improved irrigation and superior thermoconductivity), it will lead eventually to higher lesion volumes that are created under lower temperature conditions. This could possibly explain our observations of lesion structures with a multiband pattern indicating different histological characteristics.

This hypothesis on the development of inhomogeneous lesions is also supported by our findings on the mean delivered power. As our results demonstrate, the total delivered power to the tissue is significantly lower for inhomogeneous lesions as compared to homogeneous lesions, although the volume of the inhomogeneous lesions is significantly higher. As a result, a lower total amount of energy is delivered into the tissue and therefore it may create less necrotic tissue and more inhomogeneous lesions.

\section{Clinical Implications}

Previous in vitro studies on new developed ablation catheters focused on lesion volumes as a definition of catheter efficacy, which was the basis of the comparison with other catheters. In other studies, where the most ideal ablation setting was investigated for clinical practice, the focus was on lesion dimensions. It seems that previous research on the basis of catheter ablation defined lesion dimensions as the primary endpoint rather than lesion quality. Certainly, lesion volumes are very important for everyday practice, in particularly for linear ablation. However, the structure of the lesion could provide information about lesion quality in the long term.

Badger and colleagues ${ }^{16}$ studied the correlation between the amount of atrial scar and the success of initial and repeat atrial fibrillation 
ablation procedures. Their results demonstrate a discrepancy between the acute ablation endpoint of PVI and the amount of complete circumferential scarring after 3 months (6.9\% of patients). Their conclusion is that it is a difficult task to achieve long-lasting PVI, even with acute successful isolation. Furthermore, they demonstrated that differences in lesion morphology (scar patterns) could be observed during follow-up using delayedenhancement magnetic resonance imaging. ${ }^{17}$ The underlying mechanism of this acute and long-term difference could be determined by lesion quality and consistency. As our results demonstrate, there is a possibility of two types of acute lesion development with either a homogeneous or inhomogeneous structure. Our results demonstrate that SGIT catheters are associated with both larger and more inhomogeneous lesions. FGIT catheters provide more homogeneous and smaller lesions. Inhomogeneous lesions are related to significantly lower delivered power and we observed a tendency that it occurs more often using $30 \mathrm{~W}$ power output. This difference in lesion structure after acute RF ablation could have important impact on the quality and development of chronic lesions and can theoretically be responsible for the differences between acute and chronic ablation success rates. The data of Badger and colleagues ${ }^{17}$ provide indirect proof that RF lesion structures change over time and eventually result in a final scar. Our paper provides direct proof that there are differences in the histological composition of RF lesions. However, at this moment it remains unknown which combination will result in better chronic lesions. Therefore, based on our results, further evaluation is needed using a chronic model to clarify the effect of inhomogeneous lesion development.

\section{Limitations}

Based on the results of this study, some parameters showed a trend to be related to inhomogeneous lesion development, although statistical difference was not significant. An example is the distribution of homogeneous and inhomogeneous lesions for the SGIT catheter using univariate analysis. This might be related to the power of this study. Probably, statistical analysis would reach significant difference if more lesions would be included in this study. Besides, a proportion of the lesions was excluded from analysis due to the occurrence of steam pops. The unequal distribution of steam pops between the FGIT and SGIT group could interfere with the comparison of lesion volume. Due to the nature of this study, which first was a safety evaluation of the novel SGIT catheter, no sample size calculation was performed for this study. However, multivariate logistic regression analysis was performed to deal with this issue.

Based on the results it can be concluded that the SGIT catheter is a significant predictor for inhomogeneous lesion formation. However, it remains unknown whether this was caused by the new irrigation design or the metal from the catheter. At the time of this study this could not be investigated due to unavailability of certain catheters. Therefore, further evaluation of a 12-hole irrigation PtIr- and a six-hole Autip catheter is needed to really clarify the underlying element, which causes inhomogeneous lesions. Furthermore, because we found these surprising results regarding lesion structure, only two catheter types were compared in this study. It would be beneficial to compare multiple catheter types in order to better understand the underlying mechanisms of inhomogeneous lesion development. Besides, one contact force level was used during this study. Although this is a clinically relevant value, it would be interesting to evaluate the impact of different contact force values on lesion structure and quality.

In our study a canine thigh muscle preparation has been used to perform $\mathrm{RF}$ lesions. One of the advantages of this model is the flat surface of the muscle tissue. This allowed us to accurately determine lesion dimensions and continuously record tissue temperatures at different depths. However, in the trabeculated and beating endocardium, lesion formation might not be equal compared to our experiments. To really elucidate the clinical effects of different ablation catheters on lesion homogeneity these experiments should be conducted in a beating heart model as well. Furthermore, we evaluated morphological differences of lesions after $\mathrm{RF}$ catheter ablation. In order to increase the predictive clinical value, functional evaluation of the lesions would be valuable, as been performed by Datino et al. regarding the use of adenosine to evaluate pulmonary vein dormant conduction in an experimental setup. ${ }^{18}$

\section{Conclusion}

Inhomogeneous lesion developments are frequent manifestations after acute RF catheter ablation. The occurrence of inhomogeneous lesions is associated with higher lesion volumes. Furthermore, the use of an SGIT Au-tip catheter is an independent predictor for inhomogeneous lesion formation. Further studies using a chronic model are needed to evaluate the development of different lesion structures in the long term. 


\section{References}

1. Aliot EM, Stevenson WG, Almendral-Garrote JM, Bogun F, Calkins CH, Delacretaz E, Della Bella P, et al. EHRA/HRS expert consensus on catheter ablation of ventricular arrhythmias: Developed in a partnership with the European Heart Rhythm Association (EHRA), a registered branch of the European Society of Cardiology (ESC), and the Heart Rhythm Society (HRS); in collaboration with the American College of Cardiology (ACC) and the American Heart Association (AHA). Heart Rhythm 2009; 6:886-933.

2. Dorwarth U, Fiek M, Remp T, Reithmann C, Dugas M, Steinbeck G, Hoffmann E, et al. Radiofrequency catheter ablation: Different cooled and noncooled electrode systems induce specific lesion geometries and adverse effects profiles. Pacing Clin Electrophysiol 2003; 26:1438-1445.

3. Lewalter T, Weiss C, Spencker S, Jung W, Haverkamp W, Willems S, Deneke T, et al. Gold vs. platinum-iridium tip catheter for cavotricuspid isthmus ablation: The AURUM 8 study. Europace 2011; 13:102-108.

4. Balazs T, Laczko R, Bognar E, Akman S, Nagy P, Zima E, Dobranszky $\mathrm{J}$, et al. Ablation time efficiency and lesion volume: In vitro comparison of $4 \mathrm{~mm}$, non irrigated, gold- and platinum-iridiumtip radiofrequency ablation catheters. J Interv Card Electrophysiol 2013; 36:13-18.

5. Linhart M, Mollnau H, Bitzen A, Wurtz S, Schrickel JW, Andrie $\mathrm{R}$, Stockigt F, et al. In vitro comparison of platinum-iridium and gold tip electrodes: Lesion depth in $4 \mathrm{~mm}, 8 \mathrm{~mm}$, and irrigated-tip radiofrequency ablation catheters. Europace 2009; 11:565-570.

6. Linhart M, Liberman I, Schrickel JW, Mittmann-Braun EL, Andrie R, Stockigt F, Kreuz J, et al. Superiority of gold versus platinum irrigated tip catheter ablation of the pulmonary veins and the cavotricuspid isthmus: A randomized study comparing tip temperatures and cooling flow requirements. J Cardiovasc Electrophysiol 2012; 23:717-721.

7. Marrouche NF, Guenther J, Segerson NM, Daccarett M, Rittger $\mathrm{H}$, Marschang H, Schibgilla V, et al. Randomized comparison between open irrigation technology and intracardiac-echo-guided energy delivery for pulmonary vein antrum isolation: Procedural parameters, outcomes, and the effect on esophageal injury. J Cardiovasc Electrophysiol 2007; 18:583-588.

8. Akca F, Zima E, Vegh EM, Szeplaki G, Skopal J, Hubay M, Lendvai Z, et al. Radiofrequency ablation at low irrigation flow rates using a novel 12-hole gold open-irrigation catheter. Pacing Clin Electrophysiol 2013; 36:1373-1381.
9. Wittkampf FH, Nakagawa H, Foresti S, Aoyama H, Jackman WM. Saline-irrigated radiofrequency ablation electrode with external cooling. J Cardiovasc Electrophysiol 2005; 16:323-328.

10. Erdogan A, Grumbrecht S, Carlsson J, Roederich H, Schulte B, Sperzel J, Berkowitsch A, et al. Homogeneity and diameter of linear lesions induced with multipolar ablation catheters: In vitro and in vivo comparison of pulsed versus continuous radiofrequency energy delivery. J Interv Card Electrophysiol 2000; 4:655661.

11. Wittkampf FH, Nakagawa H. RF catheter ablation: Lessons on lesions. Pacing Clin Electrophysiol 2006; 29:1285-1297.

12. Nakagawa H, Yamanashi WS, Pitha JV, Arruda M, Wang X, Ohtomo $\mathrm{K}$, Beckman KJ, et al. Comparison of in vivo tissue temperature profile and lesion geometry for radiofrequency ablation with a saline-irrigated electrode versus temperature control in a canine thigh muscle preparation. Circulation 1995; 91:2264-2273.

13. Kuck KH, Reddy VY, Schmidt B, Natale A, Neuzil P, Saoudi N, Kautzner J, et al. A novel radiofrequency ablation catheter using contact force sensing: Toccata study. Heart Rhythm 2012; 9 : $18-23$.

14. Lewalter T, Bitzen A, Wurtz S, Blum R, Schlodder K, Yang A, Lickfett L, et al. Gold-tip electrodes-a new “deep lesion” technology for catheter ablation? In vitro comparison of a gold alloy versus platinum-iridium tip electrode ablation catheter. J Cardiovasc Electrophysiol 2005; 16:770-772.

15. Weiss C, Antz M, Eick O, Eshagzaiy K, Meinertz T, Willems S. Radiofrequency catheter ablation using cooled electrodes: Impact of irrigation flow rate and catheter contact pressure on lesion dimensions. Pacing Clin Electrophysiol 2002; 25:463469.

16. Badger TJ, Daccarett M, Akoum NW, Adjei-Poku YA, Burgon NS, Haslam TS, Kalvaitis S, et al. Evaluation of left atrial lesions after initial and repeat atrial fibrillation ablation: Lessons learned from delayed-enhancement MRI in repeat ablation procedures. Circ Arrhythm Electrophysiol 2010; 3:249-259.

17. Badger TJ, Oakes RS, Daccarett M, Burgon NS, Akoum N, Fish EN, Blauer JJ, et al. Temporal left atrial lesion formation after ablation of atrial fibrillation. Heart Rhythm 2009; 6:161-168.

18. Datino T, Macle L, Qi XY, Maguy A, Comtois P, Chartier D, Guerra $\mathrm{PG}$, et al. Mechanisms by which adenosine restores conduction in dormant canine pulmonary veins. Circulation 2010; 121:963972 